\title{
Aspects of Delay Diversity in OFDM
}

\author{
Gerhard Bauch \\ DoCoMo Euro-Labs \\ Landsberger Strasse 312, 80687 Munich, Germany \\ e-mail: bauch@docomolab-euro.com
}

\begin{abstract}
We consider several aspects of delay diversity in coded OFDM. The cyclic properties of the FFT allow to do delay diversity in a cyclic manner without exceeding the guard interval. We investigate the impact of different cyclic delays in terms of achievable diversity level, information theory and BER performance. Furthermore, we propose an interleaving and user assignment strategy which allows multiple users to exploit the full spatial diversity in an OFDMA system with appropriately chosen cyclic delays. Finally, we introduce a scheme with low delay and low reference symbol overhead for differential modulation in frequency direction which can be detected non-coherently and is able to cope with the increased frequency-selectivity which is caused by the cyclic delays.
\end{abstract}

\section{INTRODUCTION}

Transmit antenna diversity techniques play an important role in wireless communications for establishing a reliable link in fading environments. Besides performance, complexity and backward compatibility are essential design parameters. A very simple transmit antenna diversity method is delay diversity [1], [2]. Delay diversity techniques have regained attraction recently as simple transmit antenna diversity methods. The idea is to transmit delayed replicas of a signal simultaneously from several antennas which are separated far enough in order to provide uncorrelated fading channels. Essentially, when delay diversity is applied, the receiver sees an equivalent single-input channel with increased frequencyselectivity which can be exploited by an equalizer.

A problem associated with delay diversity is that the delays may exceed the capability of an equalizer or require a longer guard interval which means reduced bandwidth-efficiency. An elegant solution to provide delay diversity without exceeding the guard interval in orthogonal frequency division multiplex (OFDM) is to do the delays in a cyclic manner. This was proposed as cyclic delay diversity in [3] and in [4] where it was named circular generalized delay diversity. In this paper, we refer to the scheme as cyclic delay diversity. Since OFDM applies only a one tap equalizer, the increased frequency diversity needs to be exploited by a forward error correcting decoder.

Initial papers aimed to apply cyclic delay diversity in existing systems without modification of standards. E.g. [3] considered the implementation of cyclic delay diversity in terrestrial digital video broadcasting (DVB-T). The gains for different values of the cyclic delay were investigated by means of simulation. The impact of different cyclic delays on the performance is investigated in more detail in [5].

The combination of cyclic delay diversity with beamforming has been considered in [6], where several beams are used as virtual antennas over which cyclically delayed versions of the same signal are transmitted. The application of cyclic delay diversity to multi-carrier CDMA (MC-CDMA) was presented in [7].

In [8], cyclic delay diversity was analyzed with means of coding theory using the design criteria for space-time codes which where presented in [9]. Requirements for the outer FEC code and an interleaver are given which allow to obtain full diversity in frequency-flat and frequency-selective channels.

Space-frequency code construction based on cyclic delay diversity and additional shifts in frequency direction is considered in [10]. The additional shift in frequency allows to derive a detector which exploits the spatial diversity without the need for an outer FEC decoder.

In this paper, we treat various further aspects of cyclic delay diversity such as an information theoretic investigation of the optimum cyclic delays, an interleaving strategy for bit-interleaved coded modulation and multi-user aspects on Orthogonal Frequency Division Multiple Access (OFDMA), where we propose an assignment of users to subcarriers, which allows multiple users to exploit the full spatial diversity.

Furthermore, we address the application of differential modulation which can be detected non-coherently, i.e. without any channel estimation at the receiver. This is of relevance since a disadvantage of cyclic delay diversity, which is neglected in most papers, is that channel estimation becomes a more difficult task due to the increased frequency-selectivity. It has been shown in [11], [12] that with $n_{T}$ transmit antennas, the number of required pilot symbols is increased at least by a factor of $n_{T}$ compared to a single transmit antenna system, which results in lower bandwidth efficiency.

The paper is organized as follows: First, we present the channel model in Section II. The principle of cyclic delay diversity is explained in Section III before we investigate in Section IV the impact of different choices of the cyclic delay on the effective channel and the achievable diversity level. The capacity of cyclic delay diversity is derived in Section $\mathrm{V}$. The impact of particular realizations of the cyclic delay as well as the capacity gains over single transmit antenna systems are evaluated. Section VI proposes a scheme which allows multiple users to exploit spatial diversity in OFDMA. Finally, the application of differential modulation with cyclic delay diversity is proposed and evaluated in Section VII.

\section{Channel Model}

We consider an equivalent baseband multiple-input multiple-output (MIMO) channel with $n_{T}$ transmit antennas 
and $n_{R}$ receive antennas. The impulse response from transmit antenna $n$ to receive antenna $m$ at time $t$ is given by the $1 \times N_{s}$ vector

$$
\mathbf{h}_{t}^{(n m)}=\left[h_{t}^{(n m)}(0), h_{t}^{(n m)}(1), \ldots, h_{t}^{(n m)}(D), 0, \ldots, 0\right],
$$

where $D$ is the memory per subchannel from a transmit to a receive antenna and $N_{s}$ is the FFT size of the OFDM modulator. The channel coefficients $h_{t}^{(n m)}(d)$ are complex Gaussian random variables and the power delay profile is the same for all transmit antennas. The channel is assumed to be constant during transmission of an OFDM symbol such that - in case of a sufficiently long guard interval - no intercarrier interference occurs. Additive white Gaussian noise with variance $\sigma^{2}$ per real dimension is added at each receive antenna. The total transmit energy per time slot is denoted $E_{S}$, i.e. the energy per symbol transmitted from each antenna is given by $E_{S} / n_{T}$.

\section{Principle of CyClic Delay Diversity}

The principle of cyclic delay diversity is depicted in Figure 1. The data is encoded by a forward error control (FEC)

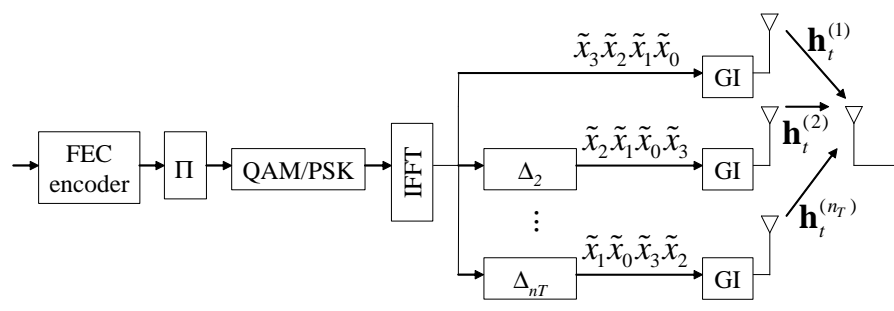

Fig. 1. Cyclic delay diversity in coded OFDM. Transmitter.

encoder. After an optional interleaver $\Pi$, the code bits are mapped e.g. on QAM or PSK symbols. OFDM is implemented using the inverse fast Fourier transform (IFFT) of size $N_{s}$, where $N_{s}$ is the number of subcarriers. The output symbols of the IFFT are denoted $\tilde{x}_{t}, t=0, \ldots, N_{s}-1$. Each antenna introduces a different cyclic delay $\Delta_{n}, n=1, \ldots, n_{T}$, i.e. the transmit symbol from antenna $n$ at time $t$ is given by

$$
x_{t}^{(n)}=\tilde{x}_{\left(t-\Delta_{n}\right)} \bmod N_{s}, t=0, \ldots, N_{s}-1, n=1, \ldots, n_{T} .
$$

Before transmission, a cyclic guard interval (GI) is included at each transmit antenna as it is usually done in OFDM.

The system is equivalent to transmission of the sequence $\tilde{\mathbf{x}}=\left[\tilde{x}_{0}, \ldots, \tilde{x}_{N_{s}-1}\right]$ over a frequency-selective channel with one transmit antenna and impulse response

$$
\mathbf{h}_{\text {equ }, t}^{(1 m)}=\left[h_{\text {equ }, t}^{(1 m)}(0), \ldots, h_{\text {equ }, t}^{(1 m)}\left(N_{s}-1\right)\right]
$$

to receive antenna $m, m=1, \ldots, n_{R}$, where with (1)

$$
h_{\text {equ }, t}^{(1 m)}(d)=\sum_{n=1}^{n_{T}} h_{t}^{(n m)}\left(\left(d-\Delta_{n}\right) \bmod N_{s}\right) .
$$

The receiver is a standard OFDM receiver which removes the guard interval and performs the FFT before QAM/PSK demapping, deinterleaving and decoding.

Basically, cyclic delay diversity has transformed the multiple-input multiple-output (MIMO) channel into a single-input multiple-output (SIMO) channel with increased frequency-selectivity, i.e. the spatial diversity is transformed into frequency diversity which can be picked up by an outer FEC decoder. In case of a convolutional code, the maximum diversity level which can be exploited by the decoder is determined by the free distance of the code.

\section{IMPACT OF The CyClic Delays on the EFFECTIVE Channel Impulse Response AND The Diversity LEVEL}

The characteristic of the resulting frequency domain channel depends on the choice of the cyclic delays $\Delta_{n}$. The channel coefficient of the $d$-th subcarrier at receive antenna $m$ in the $k$-th OFDM symbol is given by

$$
H_{k}^{(m)}(d)=\sum_{n=1}^{n_{T}} H_{k}^{(n m)}(d) e^{-j 2 \pi d \Delta_{n} / N_{s}}, \begin{aligned}
& d=0, \ldots, N_{s}-1, \\
& m=1, \ldots, n_{R},
\end{aligned}
$$

where

$$
\mathbf{H}_{k}^{(n m)}=\left[H_{k}^{(n m)}(0), \ldots, H_{k}^{(n m)}\left(N_{s}\right)\right]
$$

is the FFT of the impulse response (1).

The full spatial diversity can be exploited if [13], [14]

$$
\Delta_{n}>\Delta_{n-1}+D
$$

where $\Delta_{n}$ is normalized to the FFT sample spacing, $\Delta_{1}=0$, and $D$ is the channel memory. Since $D$ is unknown at the transmitter but the length $G$ of the guard interval represents the assumed maximum length of the impulse response, the cyclic delay could be chosen according to

$$
\Delta_{n}=G+1+\Delta_{n-1}
$$

Another save option is to choose the maximum possible cyclic delays according to

$$
\Delta_{n}=\frac{N_{s}(n-1)}{n_{T}}=\frac{N_{s}}{n_{T}}+\Delta_{n-1} .
$$

This particular choice of the cyclic delays has consequences for the effective channel frequency response: In case of $n_{T}=2$ transmit antennas and a cyclic delay of $\Delta_{2}=N_{S} / 2$, the term $e^{-j 2 \pi d \Delta_{n} / N_{s}}$ in (5) can take two different values resulting in the effective channel coefficients

$$
\begin{aligned}
& H_{k}^{(m)}(d)=H_{k}^{(1 m)}(d)+H_{k}^{(2 m)}(d), d \text { even } \\
& H_{k}^{(m)}(d)=H_{k}^{(1 m)}(d)-H_{k}^{(2 m)}(d), d \text { odd. }
\end{aligned}
$$

Given a uniform power delay profile for all transmit antennas, adjacent subcarriers happen to be uncorrelated, whereas the channel taps of every second subcarrier are correlated or even identical in case of a flat fading channel. Hence, in a flat fading channel, we observe only two different states of the effective frequency domain channel within an OFDM symbol.

In the sequel, we denote the number of different channel states which would result in a frequency-flat channel by $S$, e.g. $S=2$ for $n_{T}=2$ and $\Delta_{2}=N_{s} / 2$. I.e. the spatial diversity is transformed into frequency diversity over a well defined number of $S$ adjacent subcarriers. If we choose a cyclic delay of $\Delta_{2}=\frac{N_{s}}{4}$ with $n_{T}=2$, the term $e^{-j 2 \pi d \Delta_{n} / N_{s}}$ in (5) can take four different values, i.e. in a frequency-flat channel we 
observe four different channel states of the resulting frequency domain channel $\mathbf{H}_{k}^{(m)}$. For $\Delta_{2}=\frac{N_{s}}{8}$, we obtain 8 different states etc. Similarly, in case of $n_{T}=4$ transmit antennas and cyclic delays according to (9), we observe four different channel states resulting from (5) in an OFDM symbol.

We will exploit this property of the FFT in the following sections.

\section{Capacity of Cyclic Delay Diversity}

\section{A. Capacity Calculation}

The capacity of cyclic delay diversity can be computed from the frequency domain representation (5) of the equivalent frequency-selective single-input multiple-output (SIMO) channel. We collect the channel coefficients of the SIMO channel for subcarrier $d$ in the $n_{R} \times 1$ matrix

$$
\tilde{\mathbf{H}}_{k}(d)=\left[H_{k}^{(1)}(d), \ldots, H_{k}^{\left(n_{R}\right)}(d)\right]^{T}
$$

and assume a sufficiently long guard interval of length $G \geq D$. Then, the capacity of cyclic delay diversity with no channel state information (CSI) at the transmitter but perfect CSI at the receiver for the channel realization $\mathbf{H}_{k}$ is given by

$C\left(\mathbf{H}_{k}\right)=\frac{1}{N_{s}+G} \sum_{d=0}^{N_{s}-1} \log _{2}\left\{\operatorname{det}\left(\mathbf{I}_{n_{R}}+\frac{E_{s}}{N_{0} n_{T}} \tilde{\mathbf{H}}_{k}(d) \tilde{\mathbf{H}}_{k}(d)^{H}\right)\right\}$.

Depending on the time variance of the channel, different capacity concepts are meaningful: In an ergodic channel, the capacity is given by the expectation $C=\mathscr{E}_{\mathbf{H}_{k}}\left\{C\left(\mathbf{H}_{k}\right)\right\}$. This ergodic capacity implies that each codeword faces all possible channel states, i.e. a fast fading channel. The ergodic capacity is less suitable for the evaluation of spatial diversity techniques. The implicit time diversity provides already an infinite diversity level. Consequently, we do not see capacity advantages from additional spatial diversity. A more useful view in terms of diversity is outage capacity. This implies a slowly time varying channel where each codeword faces only one channel state. More precisely, we use a quasistatic channel model, i.e. the channel is assumed to be constant during transmission of a block and changes independently from one block to the next. The $x \%$ outage capacity $C_{x}$ is the capacity which is guaranteed in $(100-x) \%$ of the channel realizations, i.e. we expect an outage of $x \%$.

The capacity (12) implies Gaussian transmit symbols. However, in real world systems, the transmit symbols will be taken from an $M$-QAM or $M$-PSK constellation $\mathscr{C}$. Taking this restriction on the transmit symbol alphabet into consideration, the capacity or mutual information, respectively, can be computed using the general formula for the conditional mutual information [15] between the $M$-ary frequency domain transmit symbols $\mathbf{x}(d)=\left[x_{0}(d), x_{1}(d), \ldots\right]$ and received symbols $\mathbf{y}(d)$ for a particular realization $\tilde{\mathbf{H}}_{k}$ of the effective frequency domain channel:

$$
I\left(\mathbf{x}, \mathbf{y} \mid \tilde{\mathbf{H}}_{k}\right)=\frac{1}{N_{s}+G} \sum_{d=0}^{N_{s}-1}\left[H(\mathbf{x}(d))-H\left(\mathbf{x}(d) \mid \mathbf{y}(d), \tilde{\mathbf{H}}_{k}(d)\right)\right],
$$

where $H(\mathbf{x})$ and $H\left(\mathbf{x} \mid \mathbf{y}, \tilde{\mathbf{H}}_{k}\right)$ denote the entropy and the conditional entropy, respectively, and $\mathbf{x}(d), \mathbf{y}(d)$ and $\left.\tilde{\mathbf{H}}_{k}(d)\right)$ refer to the transmitted symbols, received symbols and the SIMO channel, respectively, for subcarrier $d$.

The transmit symbols $x_{t}$ are assumed to be equally likely, i.e. the entropy is given by

$$
H(\mathbf{x}(d))=\log _{2} M .
$$

The conditional entropy is obtained from

$$
\begin{array}{r}
H\left(\mathbf{x}(d) \mid \mathbf{y}(d), \tilde{\mathbf{H}}_{k}(d)\right)=-\mathscr{E}_{x_{t}(d), \mathbf{y}(d)}\left\{\log _{2} p\left(x_{t}(d) \mid \mathbf{y}(d), \tilde{\mathbf{H}}_{k}(d)\right)\right\} \\
=\mathscr{E}_{x_{t}(d), \mathbf{y}(d)}\left\{\sum_{\log _{2} \frac{\sum_{t}(d) \in \mathscr{C}}{p\left(\mathbf{y}(d) \mid x_{t}(d), \tilde{\mathbf{H}}_{k}(d)\right)}}^{p\left(\mathbf{y}(d) \mid x_{t}(d), \tilde{\mathbf{H}}_{k}(d)\right)}\right\},
\end{array}
$$

where $\mathscr{E}_{x_{t}(d), \mathbf{y}(d)}$ denotes expectation with respect to $x_{t}(d)$ and $\mathbf{y}(d)$ and

$$
p\left(\mathbf{y} \mid x_{t}, \tilde{\mathbf{H}}_{k}\right)=\frac{1}{\left(2 \pi \sigma^{2}\right)^{-n_{R}}} \exp \left(-\frac{\left\|\mathbf{y}(d)-\tilde{\mathbf{H}}_{k}(d) x_{t}(d)\right\|^{2}}{2 \sigma^{2}}\right) .
$$

Similar as in the case of capacity for Gaussian transmit symbols, we can analyse the ergodic or outage mutual information.

\section{B. Numerical Results}

In the numerical results shown below, we neglect the capacity loss due to the guard interval, i.e. we set $G=0$ in (12) and (13), in order to make comparisons easier. The capacity including a certain guard interval of length $G$ is obtained by multiplying the presented capacity curves by $N_{s} /\left(N_{s}+G\right)$. Unless stated otherwise, we show results for $N_{s}=64$ subcarriers. Throughout this paper, we often consider frequency-flat channels first in order to isolate the spatial effects before giving results for frequency-selective channels.

1) Capacity Impact of the Choice of the Cyclic Delays: We first investigate the impact of the cyclic delay on the capacity. Figure 2 shows the $1 \%$ outage capacity for $n_{T}=2$ transmit antennas in a frequency-flat channel with Gaussian transmit symbols. For $n_{R}=1$ receive antenna, all cyclic delays yield the same capacity except for $\Delta_{2}=N_{s} / 2=32$ which shows a slight degradation. For $n_{R}=2$ receive antennas, this effect vanishes. For comparison, we also included the capacity of the channel itself both for one and two transmit antennas. In case of $n_{R}=1$, cyclic delay diversity almost achieves the $n_{T}=2$ channel capacity and shows a significant diversity advantage over a single transmit antenna system. For $n_{R}=2$, there is still a clear diversity gain over a single transmit antenna system. However, since transmit diversity schemes cannot reach the channel capacity in case of multiple receive antennas [16], [17], there is a significant degradation compared to the $n_{T}=2$ channel capacity which results from the different asymptotic slopes of the capacity curves.

Figure 3 shows the $1 \%$ outage capacity for BPSK and QPSK modulation, respectively. The cyclic delay appears to have a significant impact on the capacity, particularly for $n_{R}=1$ receive antenna. E.g. $\Delta_{2}=N_{s} / 2=32$ is optimum only for code 


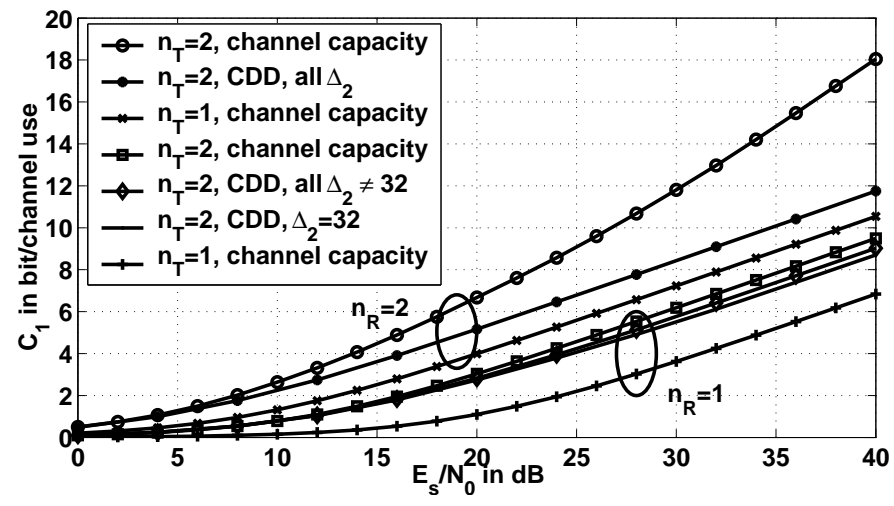

Fig. 2. $1 \%$ outage capacity. Frequency-flat channel, Gaussian transmit symbols, $N_{s}=64$.

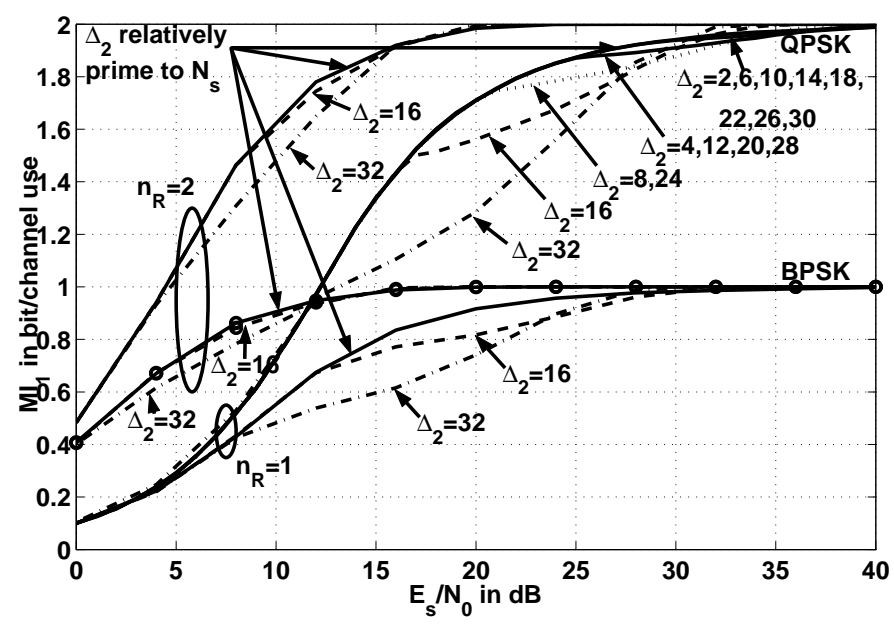

Fig. 3. $1 \%$ outage capacity. Frequency-flat channel, BPSK and QPSK, $n_{T}=2$, $N_{s}=64$.

rates $R<1 / 2$, i.e. a mutual information of 0.5 or $1 \mathrm{bit} / \mathrm{channel}$ use for BPSK or QPSK, respectively.

This can be explained as follows: If the original channel was frequency flat and the cyclic delay is not relatively prime to the number $N_{S}$ of subcarriers, the frequency domain channel coefficients $H_{k}^{(m)}(d)$ of the effective channel according to (5) can take a limited number $S$ of distinct states as explained in Section IV. Figure 3 shows the capacity for $n_{T}=2$ transmit antennas and different values of the cyclic delay which result in different values of $S$. It turns out, that the optimality of the cyclic delay depends on the code rate. For higher code rates, a higher value of $S$ is required for optimum performance. Interestingly, for very high code rates, $S=2$ becomes optimum again.

An intuitive - not theoretically exact - explanation is as follows: Consider a systematic code of rate $R$ and a cyclic delay which leads to $S=2$, e.g. $\Delta_{2}=N_{S} / 2$. Since there are only 2 different channel states, it can happen that every other subcarrier is in a very bad state and the symbols transmitted on those subcarriers are essentially punctured. Now, if the code rate is higher than $1 / 2$, i.e. $R \geq 1 / 2$, e.g. all parity bits plus some systematic bits are punctured and the code cannot be detected error free. Similarly, in case of $S=4$, a systematic code of rate $R>3 / 4$ is not suitable. We observed similar but not that clear behaviour for more transmit antennas [18].

Note that even though we gave examples for BPSK and QPSK modulation, the aforementioned effects are independent of the particular modulation method. In contrast to [5], we found no dependence of the optimum cyclic delays on the modulation alphabet but rather on the code rate. Due to a higher diversity level, the aforementioned effects are reduced if multiple receive antennas are applied. The same is true for frequency-selective channels, where we found that all cyclic delays which meet $\Delta_{n}>\Delta_{n-1}+D$ show similar performance. For frequency-selective channels, we also investigated different FFT sizes $\left(N_{s}=64,128,512,1024\right)$ and found no dependence of the capacity results on the number of subcarriers given that the cyclic delays are chosen as the same fraction of $N_{s}$.

2) Capacity Gain of Cyclic Delay Diversity Over Single Transmit Antenna Systems: Finally, we investigate how much we can gain in terms of capacity using cyclic delay diversity in frequency-selective channels. Frequency-selective channels already provide a significant amount of frequency diversity. Consequently, the gain obtained by additional spatial diversity decreases with increasing level of frequency diversity.

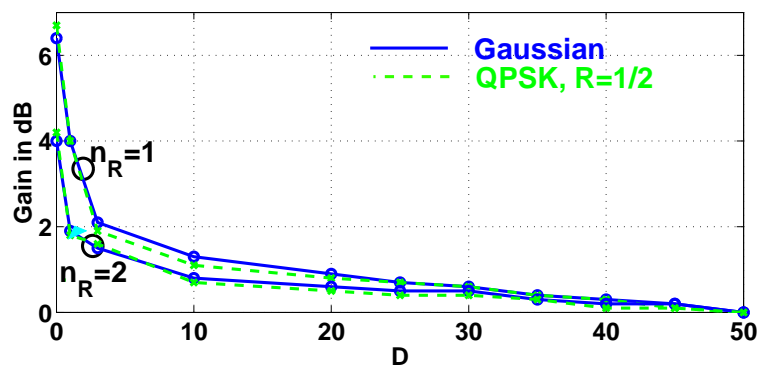

Fig. 4. $1 \%$ outage capacity gain of cyclic delay diversity over a single transmit antenna system. Frequency-selective channels with uniform power delay profile, $n_{T}=2, N_{s}=64$.

Figure 4 depicts the gain of cyclic delay diversity over a single transmit antenna system in a frequency-selective channel with uniform power delay profile depending on the number $D+1$ of channel taps. We show the asymptotic gain for Gaussian transmit symbols as well as the gain for QPSK at a code rate $R=1 / 2$, which turns out to be almost identical. As expected, the gains are reduced if additional receive antenna diversity is available. It can be concluded that cyclic delay diversity provides reasonable gains up to a frequency diversity of about 30 independent taps.

\section{Cyclic Delay Diversity in OFDMA}

\section{A. Assignment of Users to Subcarriers and Interleaving}

The properties of the resulting equivalent channel which were mentioned in Section IV have consequences for the interleaver design and for the allocation of multiple users to subcarriers in an orthogonal frequency division multiple access (OFDMA) system. Our goal is to guarantee that each user can 
pick up the full spatial diversity. In order to achieve this, we make use of the finding of Section IV that with cyclic delays which are not relatively prime to $N_{s}$, the spatial diversity is distributed over a defined block of $S$ adjacent subcarriers. For simplicity, we assume code rates $R \leq 0.5$ in the following, i.e. the cyclic delays are chosen according to (9. For higher code rates, the respective cyclic delays have to be adapted as explained in Section V-B.1.

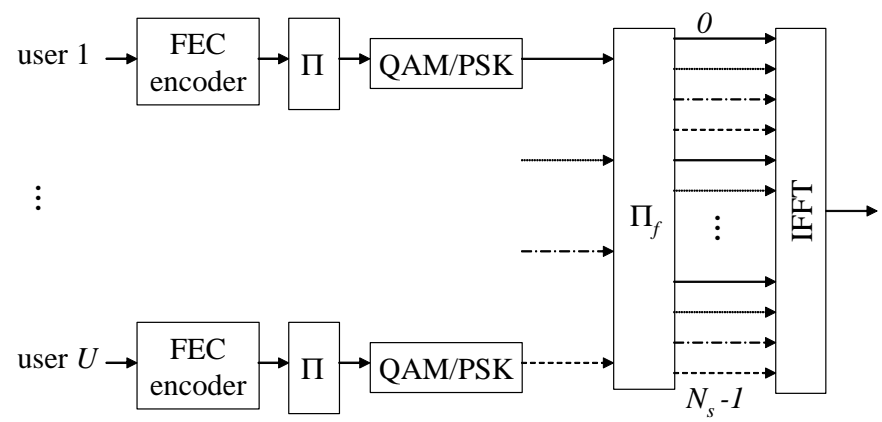

Fig. 5. OFDMA with block frequency interleaver $\Pi_{f}$.

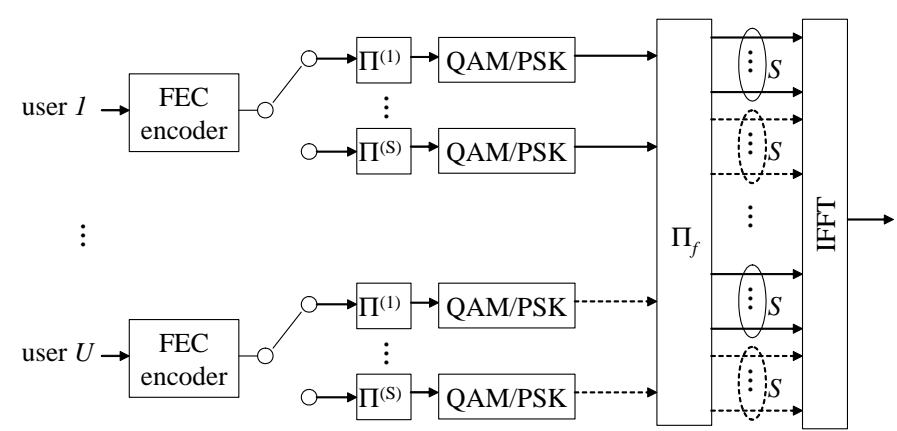

Fig. 6. OFDMA with multi-stream interleaving $\Pi^{(s)}$ and group frequency interleaver $\Pi_{f}$.

In a conventional OFDMA system, the subcarriers which are allocated to a particular user are either a set of adjacent subcarriers or a block frequency interleaver distributes the symbols of a user on subcarriers with a fixed spacing in order to exploit frequency diversity. If cyclic delays according to (9) are applied, such a block interleaving may result in a complete failure to exploit spatial diversity. The problem is illuminated in Figure 5 for the example of $U=4$ users. The subcarriers $d=0,4,8, \ldots$ are allocated to user 1 . However, as explained above, user 1 can pick up spatial diversity only if neighbouring subcarriers are allocated to him. Therefore, we propose to allocate groups of $S$ neighbouring subcarriers to each user as indicated in Figure 6. An additional groupwise frequency interleaving $\Pi_{f}$ enables exploitation of frequency diversity which is available due to the frequency selectivity of the channel. Since with cyclic delays according to (9), the spatial diversity is transformed into $S$ adjacent subcarriers, we can assign up to $\left\lfloor N_{S} / S\right\rfloor$ users in an OFDM symbol and provide full spatial diversity for each user, where $\lfloor. \mid$ indicates the floor function. However, in frequency-selective channels, a smaller number of users should be assigned to each OFDM symbol in order to allow also the exploitation of frequency diversity.
Furthermore, the choice of the cyclic delay should also be taken into consideration in the design of the bit interleavers $\Pi$. A convolutional code picks up the maximum diversity if successive code bits are transmitted via uncorrelated subcarriers. Therefore, we propose to split the stream of code bits into $S$ streams. The bits in each stream are optionally interleaved and mapped on constellation elements of a modulation scheme.

The complete transmitter is depicted in Figure 6. The bits of each user are encoded by a forward error control code. A serial to parallel converter splits the stream of code bits into $S$ streams. The first bit goes to stream 1, the second to stream 2 etc.. Within each stream, an optional bit interleaver $\Pi^{(s)}$ permutes the code bits before mapping them to constellation elements of a digital modulation method such as QAM or PSK. The modulation symbols can optionally be interleaved. Then, the $S$ streams are mapped on groups of $S$ neighbouring subcarriers, where stream $s$ always goes on the $s$-th subcarrier within a group. The spacing between groups of a particular user is fixed, i.e. we apply a groupwise frequency block interleaver $\Pi_{f}$. In this way, the frequency diversity of the original channel is obtained by distributing groups over the entire bandwidth whereas spatial diversity is exploited by the streams within one group which are transmitted on neighbouring subcarriers. Optionally, the assignment $\Pi_{f}$ can be changed from one OFDM symbol to the next. I.e., subcarriers $0, \ldots, S-1$ can be assigned to user 1 in the first OFDM symbol but to user 2 in the second OFDM symbol. The receiver performs the reverse interleaving operations of the transmitter.

\section{B. Simulation Results}

For the simulation results presented in this section, we restrict ourselves to BPSK modulation since we found that in principle the results are independent of the particular modulation format. We apply a convolutional code with rate $R=1 / 2$, constraint length 3 , and free distance $d_{f}=5$ for channel coding. The FFT size is $N_{s}=64$ subcarriers. We assume a spatially uncorrelated channel with $n_{T}=2$ transmit and $n_{R}=1$ receive antennas and quasistatic fading, i.e. the channel coefficients are constant during transmission of a coded frame and change independently from one frame to the next. In principle, the results hold true also for more antennas. First, we present results for flat fading channels in order to isolate the spatial effects. Later, we consider also frequency-selective channels with $D+1$ sample-spaced taps of equal mean power where additional frequency diversity is available. In case of frequency-selective channels, we assume a sufficiently long guard interval.

1) Impact of the Cyclic Delay: In Sections IV and VI-A we proposed to choose the cyclic delay according to (9) such that adjacent subcarriers are uncorrelated and multiple users can exploit the spatial diversity. Simulation results on the frame error rate (FER) in a flat fading channel are depicted in Figure 7 for different values $\Delta_{2}$ of the cyclic delay.

The results demonstrate the superiority of the choice $\Delta_{2}=$ $N_{s} / 2=32$ according to (9), where the interleaving strategy with $S=2$ streams as proposed in Section VI has been applied. From the slope of the FER curve it can be observed that the 
full spatial diversity order of $n_{T} n_{R}=2$ is exploited. Whereas other delays $\Delta_{2}$ show almost identical performance, there is a $1 \mathrm{~dB}$ gain with $\Delta_{2}=32$.

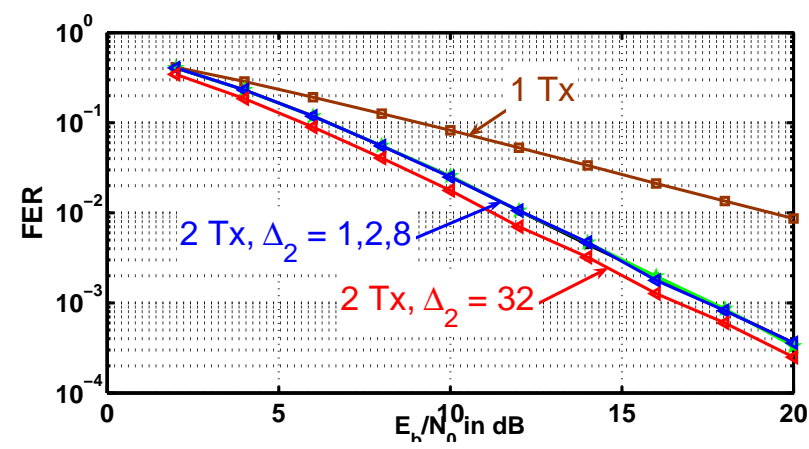

Fig. 7. $2 \mathrm{Tx}, 1 \mathrm{Rx}$ antenna, $N_{s}=64$, BPSK, quasistatic flat fading.

In the bit error rate (BER) performance, we observed hardly any performance difference for different delays in flat fading channels. The cyclic delay $\Delta_{2}=N_{s} / 2$ according to (9) performed even a little bit worse than smaller cyclic delays. This can be explained by the fact that for $\Delta_{2}=N_{s} / 2$ we have only 2 independently fading channel states, one for the even subcarriers, the other for the odd subcarriers. If one of the 2 channels happens to be in a very bad state, every other codebit of the rate $1 / 2$ convolutional code is essentially punctured by the channel. Therefore, the BER in those frames will be very high and will dominate the average BER over all simulated frames. On the other hand, a frame with a highy number of bit errors has the same impact on the FER as a frame with only one bit error. Consequently, the proposed choice (9) of the cyclic delay is optimized for FER performance.

The aforementioned results hold true also in frequencyselective channels. However, the effect of choosing different cyclic delays is reduced since also frequency diversity is available.

2) Interleaving and User Assignment: Figure 8 illuminates the effect of the multiple access and interleaving scheme according to Figure 6 for a cyclic delay of $\Delta_{2}=N_{s} / 2=32$ in both flat and frequency-selective channels. Due to the choice of the cyclic delay, all even subcarriers as well as all odd subcarriers are correlated whereas adjacent subcarriers are uncorrelated. We assume an even number $U$ of users. The BER depicted in Figure 8 is the same for each user.

First we consider a block frequency interleaver $\Pi_{f}$ as indicated in Figure 5. Since the block frequency interleaver maps all symbols of a user to either only even subcarriers or only odd subcarriers, no spatial diversity can be exploited by the FEC decoders. I.e. OFDMA with a block frequency interleaver $\Pi_{f}$ fails to exploit the spatial diversity.

In order to pick up spatial diversity, each user has to code through even and odd subcarriers. This is achieved by a groupwise frequency interleaving $\Pi_{f}$ as indicated in Figure 6.

Furthermore, it can be observed that the full spatial diversity is only obtained when the code bits are split into $S=2$ streams before bit-interleaving ( 2 branch interleaving $\Pi^{(s)}$, see Figure

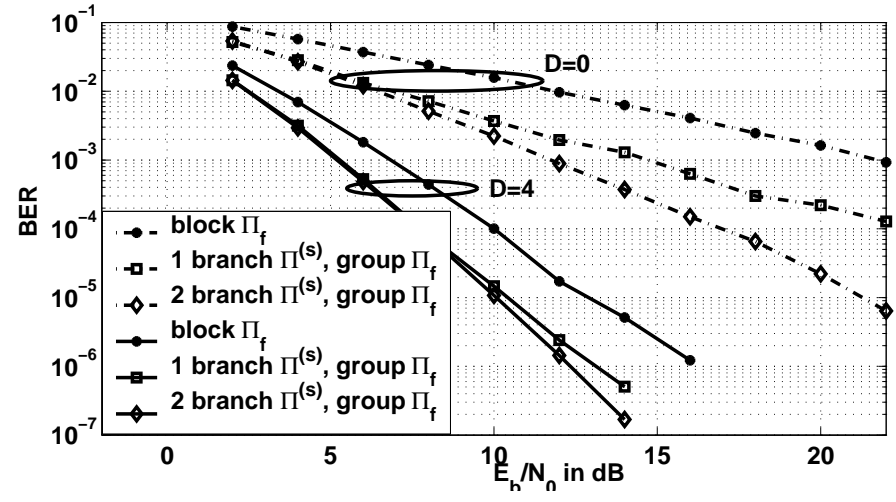

Fig. 8. 2Tx, $1 \mathrm{Rx}$ antenna, $N_{s}=64, \Delta_{2}=32$, BPSK, quasistatic flat $(D=0)$ and frequency selective $(D=4)$ fading.

6). This still holds true in a frequency selective channel. However, due to available frequency diversity, the effect is reduced.

\section{CyCliC DELAy DiVERSITY WITH DifFERENTIAL MODULATION}

Up to now, we have assumed coherent detection with perfect channel estimation. However, the increased frequencyselectivity due to the cyclic delays makes channel estimation a more difficult task than in a single transmit antenna system. Channel estimation for cyclic delay diversity has been investigated in [11], where it has been shown that with $n_{T}$ transmit antennas, the number of required pilot symbols is increased at least by a factor of $n_{T}$ compared to a single transmit antenna system, which results in lower bandwidth efficiency. Therefore, differential modulation which does not require channel estimation might be attractive. Throughout this paper, we consider a simple differential detector which takes into account two successively received symbols and requires the channel to be about constant during transmission of those two symbols. The performance might be improved by more complex multiple symble detectors [19]. We start with some general remarks on differential modulation in OFDM in Section VII-A before we propose a solution for problems which appear with cyclic delay diversity in Section VII-B.

\section{A. Differential Modulation in OFDM}

In OFDM, differential modulation can be done separately on each subcarrier given that the channel coherence time is longer than two OFDM symbol durations. However, a reference symbol has to be sent in advance on each subcarrier which results in a full OFDM symbol overhead, i.e. $N_{s}$ PSK symbols. This is undesired particularly in a $4 \mathrm{G}$ mobile radio system where we expect a large number of subcarriers but a relatively small number of OFDM symbols per frame.

An alternative is to do the differential encoding in frequency direction which requires only a single reference PSK symbol. In this case, the coherence bandwidth is required to be larger than two subcarrier spacings. This requirement might become critical with cyclic delay diversity since the diversity effect 
comes from increased frequency-selectivity and hence reduced coherence bandwidth. Particularly, in case of cyclic delays according to (9), adjacent subcarriers are uncorrelated and, therefore, straight forward differential modulation in frequency direction will fail. In the sequel, we will analyze the effect of different cyclic delays and particularly propose a solution for cyclic delays according to (9).

\section{B. Differential Modulation and Cyclic Delay Diversity}

We consider two options for the application of differential modulation in combination with cyclic delay diversity: A straight forward solution is to choose the cyclic delays according to (8), i.e. larger than the guard interval, in order to meet the full spatial diversity criterion (7) and to perform standard differential modulation in frequency direction. In order to allow simple non-coherent detection, the coherence bandwidth of the effective channel (5) should be larger than two subcarrier spacings. This might become critical since cyclic delay diversity increases the frequency-selectivity of the channel.

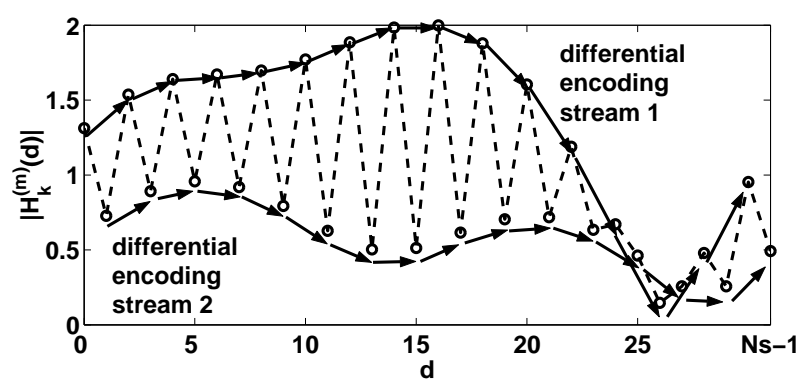

Fig. 9. Differential encoding with two streams for cyclic delay diversity with $n_{T}=2$ transmit antennas and cyclic delay $\Delta_{2}=\frac{N_{s}}{2}$. Frequency-selective channel.

Therefore, we propose another option: We propose to split the data into $S$ streams, where $S$ is the number of different states of the effective frequency domain channel which would result from (5) in a frequency-flat original channel. Separate differential modulation is performed in each of the $S$ streams. Each stream is then transmitted over subcarriers with high correlation.

An example for $n_{T}=2$ transmit antennas is given in Figure 9. According to (10), we have $S=2$. One stream is transmitted over the even numbered subcarriers, the other stream is transmitted over the odd numbered subcarriers. The (cheap) price we pay is one additional reference symbol which is required for the second stream. The transmitter of our proposed scheme is depicted in Figure 10. For non-coherent detection, the effective channel needs to be constant over two subcarriers within each stream. I.e. the original channel has to be constant over three subcarriers.

The assignment of transmit symbols to subcarriers can be realized by a block interleaver with interleaving depth $S$, i.e. an $S \times N_{S} / S$ matrix in which we write rowwise and from which we read columnwise. OFDM modulation and cyclic delay diversity are then performed as described in Section III.

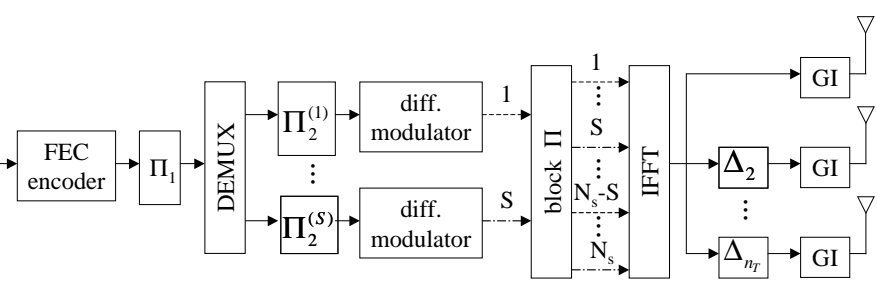

Fig. 10. Transmitter for cyclic delay diversity with multi-stream differential modulation.

The receiver is equipped with $n_{R}$ antennas. After removing the guard interval, the FFT is taken at each receive antenna. The block deinterleaver $\Pi^{-1}$ assigns the $n_{R}$ received signals of each stream to the differential detectors, which also work as receive diversity combiners. After deinterleaving according to $\Pi_{2}^{(s)-1}$ and/or $\Pi_{1}^{-1}$, an FEC decoder picks up the frequency diversity and delivers soft or hard decisions on the information bits.

In order to exploit the full available diversity and coding gain, the differential detectors should deliver soft outputs. The problem is that even in flat fading channels, the streams face different channel states and, therefore, the weighting of the decisions in different channel states is essential. Actually, this would require channel state information. A soft-output detector for DPSK with receive diversity, which does neither require knowledge of the channel coefficients nor of the noise variance is derived in [20].

1) Simulation Results: In this section, we present simulation results for a quasistatic spatially uncorrelated channel with $n_{T}=2$ transmit and $n_{R}=1$ receive antenna, i.e. the channel is constant in time over one FEC coded block and changes independently from one block to the next.

First, in Figure 11 we demonstrate the effect of multi-stream differential modulation and the impact of the interleaving scheme for a flat fading channel, DQPSK modulation, $N_{s}=64$ subcarriers and a block size of 122 uncoded bits. We use a rate $1 / 2$ convolutional code with constraint length 5 for FEC coding.

In Figure 11, we also included the BER for a single transmit antenna system with differential modulation. Using cyclic delay diversity with $\Delta_{2}=1$ yields the expected diversity level of 2 . With $\Delta_{2}=32$ and $S=1$ stream, detection fails completely since the effective channel taps of adjacent subcarriers are uncorrelated. However, we can pick up diversity by splitting the data stream into $S=2$ streams.

The impact of the interleaving strategy can be observed from Figure 10. For the same reasons as explained in Section VIB.2, Figure 8, per stream interleaving $\Pi_{2}^{(s)}$ instead of a single random interleaver $\Pi_{1}$ is essential in order to pick up the full diversity with convolutional codes of limited contraint length..

For the evaluation in frequency-selective channels, we consider a uniform power delay profile with $D+1$ sample spaced taps per subchannel, $N_{s}=2048$ subcarriers and DQPSK modulation. FEC coding is done over one OFDM symbol using a rate $1 / 2$ convolutional code with constraint length 3 .

Figure 12 shows the BER for different lengths $D$ of the channel impuls response. We compare cyclic delays of $\Delta_{2}=$ 
$D+1$ with single stream differential modulation to our 2stream proposal with a cyclic delay of $\Delta_{2}=N_{s} / 2=1024$. It can be observed that both options show similar performance. Therefore, the question arises, when our 2-stream proposal is advantageous. To answer this question, we plot the coherence

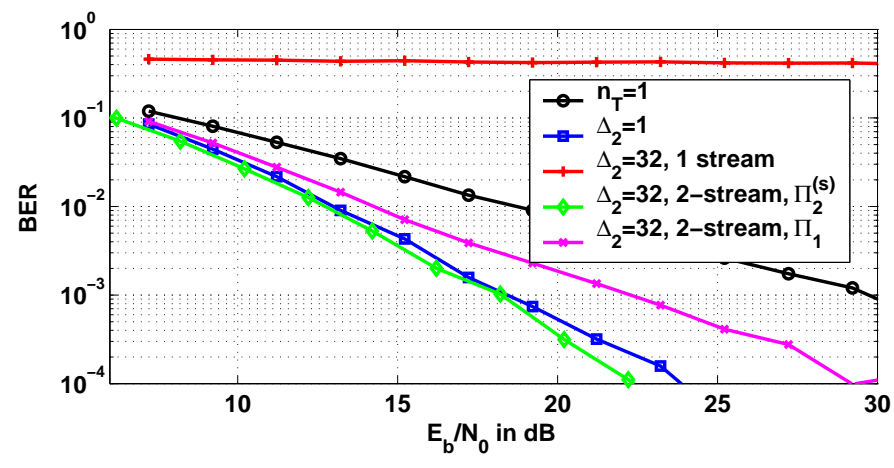

Fig. 11. Flat fading channel, $n_{T}=2, n_{R}=1$, DQPSK, $N_{s}=64$.

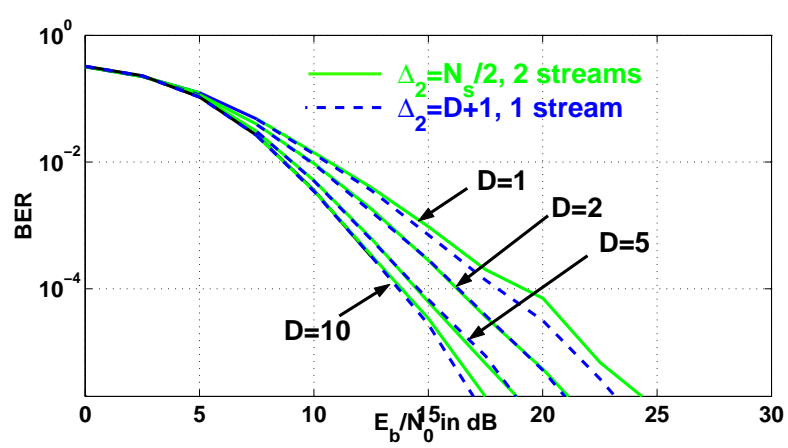

Fig. 12. Frequency-selective channels with uniform power delay profile, $D+1$ taps, $n_{T}=2, n_{R}=1$, DQPSK, $N_{s}=2048$.

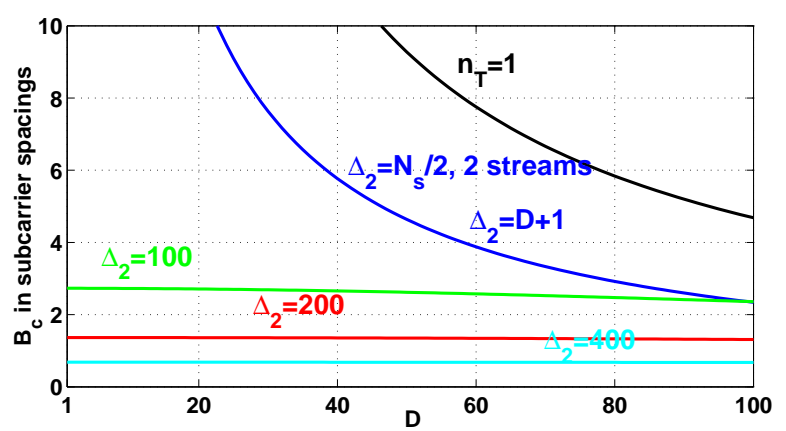

Fig. 13. Coherence bandwidth of the effective SIMO channel with cyclic delay diversity in subcarrier spacings. Frequency-selective channels with uniform power delay profile, $D+1$ taps, $N_{s}=2048$ subcarriers, $n_{T}=2$ transmit antennas.

bandwidth of the effective SIMO channel for different cyclic delays in Figure 13 as a performance measure for differential modulation. We use

$$
B_{c}=\frac{1}{15 \sigma_{\tau}}
$$

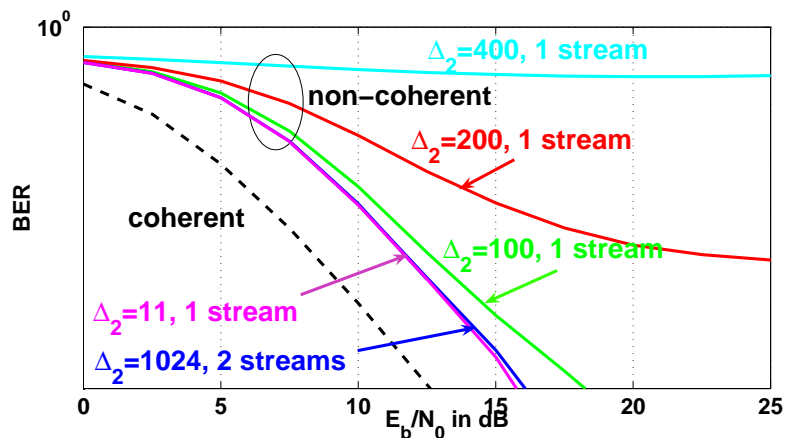

Fig. 14. Frequency-selective channel with uniform power delay profile, $D=$ 10 taps, $n_{T}=2, n_{R}=1$, DQPSK, $N_{s}=2048$.

as a rule of thumb definition for the coherence bandwidth, where $\sigma_{\tau}$ is the rms delay spread of the channel.

It can be observed that the coherence bandwidth is decreased by cyclic delay diversity compared to a single transmit antenna system. Using a cyclic delay $\Delta_{2}=D+1$ with single stream differential modulation and our proposal $\Delta_{2}=N_{s} / 2$ with 2-stream differential modulation yield virtually the same result, where in the latter case we measure the relevant coherence bandwith within each stream. This explains that both options show comparable performance in Figure 12. However, setting the cyclic delay to $\Delta_{2}=D+1$ requires knowledge of the instantaneous length of the channel impulse response at the transmitter. In general, this knowledge will not be available. The guard interval $G$ will be designed longer than the maximum expected length of the channel impulse respons. Typical values are $10-20 \%$ of the OFDM symbol duration. The transmitter can then choose the cyclic delay according to $\Delta_{2}=G+1 \geq D+1$. If the instantaneous channel impulse response is shorter than the guard interval, i.e. $D<G$, the coherence bandwidth of the effective channel will be mainly determined by the cyclic delay $\Delta_{2}$. Examples for guard intervals of 5-20\% of the OFDM symbol duration are included in Figure $13\left(\Delta_{2}=100,200,400\right)$.

It can be concluded that our proposal of 2-stream differential modulation yields the same performance as single stream differential modulation if the instantaneous channel impulse response is as long as the guard interval, but is superior if the instantaneous channel impulse response is shorter than the guard interval. These considerations are confirmed by the respective BER results in Figure 14, where for comparison we included the performance of coherent QPSK which yields the usual $3 \mathrm{~dB}$ advantage over differential modulation.

\section{CONCLUSIONS}

We considered several aspects of cyclic delay diversity as a simple and powerful transmit antenna diversity method for OFDM which enables substantial diversity gains even in relatively strong frequency-selective channels. We discussed the impact of the choice of the cyclic delays on the achievable diversity level and for enabling multiple users to pick up the full spatial diversity in OFDMA. We derived the capacity of cyclic delay diversity and showed that the optimum cyclic 
delay depend on the code rate but not on the modulation alphabet. We propose to choose the cyclic delays at different antennas realively prime which yields close to optimum performance.

Furthermore, we have presented a scheme for differential modulation in frequency direction in OFDM with cyclic delay diversity which can cope with the increased frequencyselectivity. Our multi-stream proposal yields superior performance compared to straight forward single-stream differential modulation in the likely case that the instantaneous channel impulse response is shorter than the guard interval and comparable performance if the instantaneous channel impulse response is as long as the guard interval.

Further studies which are not reported in this paper compared cyclic delay diversity to orthogonal space-time block codes as a well established transmit diversity scheme which in contrast to cyclic delay diversity performs maximum ratio combining. In case of 2 transmit and 1 receive antenna, orthogonal space-time block codes outperform cyclic delay diversity by about $1.5-1.7 \mathrm{~dB}$ both in frequency-flat and frequencyselective channels. For more than 2 transmit antennas, cyclic delay diversity shows higher capacity since in contrast to orthogonal space-time block codes it does not suffer from a rate loss.

\section{REFERENCES}

[1] A. Wittneben, "A new bandwidth efficient transmit antenna modulation diversity scheme for linear digital modulation," in International Conference on Communications (ICC), pp. 1630-1633, IEEE, 1993.

[2] N. Seshadri and J. H. Winters, "Two signaling schemes for improving the error performance of frequency-division-duplex (fdd) transmission systems using transmitter antenna diversity," International Journal of Wireless Information Networks, vol. 1, no. 1, pp. 49-59, 1994.

[3] A. Dammann and S. Kaiser, "Standard conformable antenna diversity techniques for OFDM systems and its application to the DVB-T system," in IEEE Globecom, pp. 3100-3105, November 2001.

[4] D. Gore, S. Sandhu, and A. Paulraj, "Delay diversity codes for frequency selective channels," in International Conference on Communications (ICC), pp. 1949-1953, IEEE, April 2002.

[5] M. Bossert, A. Huebner, F. Schuehlein, H. Haas, and E. Costa, "On cyclic delay diversity in OFDM based transmission schemes," in OFDM Workshop, 2002.

[6] A. Dammann, R. Raulefs, and S. Kaiser, "Beamforming in combination with space-time diversity for broadband OFDM systems," in IEEE International Conference on Communications (ICC), pp. 165-171, April 2002.

[7] A. Dammann, R. Raulefs, G. Auer, and G. Bauch, "Comparison of space-time block coding and cyclic delay diversity for a broadband mobile radio air interface," in International Symposium on Wireless Personal Multimedia Communications (WPMC), October 2003.

[8] J. Tan and G. Stuber, "Multicarrier delay diversity modulation," in IEEE Globecom Conference, December 2003.

[9] V. Tarokh, N. Seshadri, and A. Calderbank, "Space-time codes for high data rate wireless communication: Performance criterion and code construction," IEEE Transactions on Information Theory, vol. 44, pp. 744765, March 1998.

[10] A. Huebner, F. Schühlein, M. Bossert, E. Costa, and H. Haas, "On space-frequency coding using cyclic delay diversity for OFDM-based transmission systems," European Transactions on Telecommununication (ETT), vol. 14, pp. 491-500, November/December 2003.

[11] G. Auer, "Channel estimation for OFDM with cyclic delay diversity," in IEEE International Symposium on Personal, Indoor and Mobile Radio Communications (PIMRC), September 2004.

[12] G. Auer, "Channel estimation by set partitioning for OFDM with cyclic delay diversity," in IEEE Vehicular Technology Conference (VTC), September 2004.
[13] J. Winters, "The diversity gain of transmit diversity in wireless fading systems with Rayleigh fading," IEEE Transactions on Vehicular Technology, vol. 47, pp. 119-123, February 1998.

[14] G. Bauch and J. Malik, "Parameter optimization, interleaving and multiple access in OFDM with cyclic delay diversity," in IEEE Vehicular Technology Conference (VTC), May 2004.

[15] T. Cover and J. Thomas, Elements of Information Theory. New York: John Wiley \& Sons, Inc., 2nd edition ed., 1991.

[16] S. Sandhu and A. Paulraj, "Space-time block codes: a capacity perspective," IEEE Communications Letters, vol. 4, pp. 384-386, December 2000.

[17] G. Bauch, J. Hagenauer, and N. Seshadri, "Turbo processing in transmit antenna diversity systems," Annals of Telecommunications, Special Issue: Turbo codes - a widespreading technique, vol. 56, pp. 455-471, August 2001.

[18] G. Bauch, "Capacity optimization of cyclic delay diversity," in IEEE Vehicular Technology Conference (VTC), September 2004.

[19] D. Divsalar and M. Simon, "Multiple-symbol differential detection of MPSK," IEEE Transactions on Communications, vol. 38, pp. 300-308, March 1990.

[20] G. Bauch, "Multi-stream differential modulation for cyclic delay diversity in OFDM," in IEEE International Conference on Communications (ICC), June 2004.

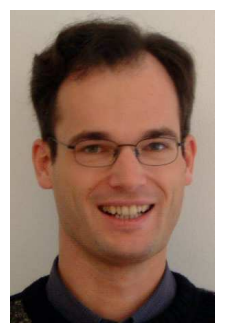

Gerhard Bauch received the Dipl.-Ing. and Dr.Ing. degree in Electrical Engineering from Munich University of Technology in 1995 and 2001, respectively, and the Diplom-Volkswirt degree from FernUniversität Hagen, Germany, in 2002. In 1996, he was with the German Aerospace Center (DLR), Oberpfaffenhofen, Germany. From 1996-2001 he was member of scientific staff at Munich University of Technology (TUM). In 1998 and 1999 he was visiting researcher at AT\&T Labs Research, Florham Park, NJ, USA. In 2002 he joined DoCoMo EuroLabs, Munich, Germany, where he is currently manager of the Advanced Radio Transmission Group. Since October 2003 he has also been an adjunct professor at Munich University of Technology (TUM). He received the best paper award of the European Personal Mobile Communications Conference (EPMCC) 1997, the Texas Instruments Award of TUM 2001 and the award of the German Information Technology Society (ITG in VDE) 2002 (ITG Förderpreis). His research interests include channel coding, turbo processing, multihop transmission, ad-hoc networks and various aspects of signal processing in multi-antenna systems (MIMO). 